\title{
THE AVERAGE ORDER OF A PERMUTATION
}

\author{
RICHARD STONG \\ Department of Mathematics \\ Rice University \\ Houston, TX 77005 \\ stong@math.rice.edu
}

Submitted: May 11, 1998; Accepted: June 23, 1998

Abstract. We show that the average order $\mu_{n}$ of a permutation in $S_{n}$ satisfies

$$
\log \mu_{n}=C \sqrt{\frac{n}{\log n}}+O\left(\frac{\sqrt{n} \log \log n}{\log n}\right)
$$

which refines earlier results of Erdős and Turán, Schmutz, and Goh and Schmutz.

\section{Introduction.}

For $\sigma \in S_{n}$ let $N(\sigma)$ be the order of $\sigma$ in the group $S_{n}$. Erdős and Turán [2] showed that if one chooses a permutation uniformly at random from $S_{n}$ then for $n$ large $\log N(\sigma)$ is asymptotically normal with mean $\left(\log ^{2} n\right) / 2$ and variance $\left(\log ^{3} n\right) / 3$. Define the average order of an element of $S_{n}$ to be

$$
\mu_{n}=\frac{1}{n !} \sum_{\sigma \in S_{n}} N(\sigma)
$$

It turns out that $\log \mu_{n}$ is much larger than $\left(\log ^{2} n\right) / 2$, being dominated by the contribution of a relatively small number of permutations of very high order. This was first shown by Erdős and Turán [3], who showed that $\log \mu_{n}=\mathbf{O}(\sqrt{n / \log n})$. This result was sharpened by Schmutz [6], and later by Goh and Schmutz [4] to show that $\log \mu_{n} \sim C \sqrt{n / \log n}$, for an explicit constant $C$. The purpose of this note is to show that

$$
\log \mu_{n}=C \sqrt{\frac{n}{\log n}}+\mathbf{O}\left(\frac{\sqrt{n} \log \log n}{\log n}\right),
$$

where $C=2.99047 \ldots$ is an explicit constant defined below. Our argument shares some similarities with that of [4], but is more elementary and permits a more explicit

1991 Mathematics Subject Classification. Primary 11N37. 
bound on the error term. The proof will be divided into three steps. First we will give upper and lower bounds on $\mu_{n}$ involving the coefficients of a certain power series, then we will use a Tauberian theorem to bound these coefficients.

For a partition $\lambda=\left(\lambda_{1}, \lambda_{2}, \ldots, \lambda_{s}\right)$ let $c_{i}(\lambda)$ be the number of parts of $\lambda$ of size $i$, let $|\lambda|=\lambda_{1}+\lambda_{2}+\cdots+\lambda_{s}$ and let $m(\lambda)=$ l.c.m. $\left(\lambda_{1}, \lambda_{2}, \ldots, \lambda_{s}\right)$. We will say that $\lambda$ is a partition of $|\lambda|$. By a sub-partition of $\lambda$ we will mean any subset of $\left(\lambda_{1}, \lambda_{2}, \ldots, \lambda_{s}\right)$ viewed as a partition of some smaller number. Then

$$
\mu_{n}=\sum_{|\lambda|=n} \frac{m(\lambda)}{1^{c_{1}(\lambda)} 2^{c_{2}(\lambda)} \ldots c_{1}(\lambda) ! c_{2}(\lambda) ! \ldots} .
$$

\section{The Upper Bound.}

Call a partition $\pi=\left(\pi_{1}, \pi_{2}, \ldots, \pi_{s}\right)$ minimal if for each sub-partition $\pi^{\prime}$ of $\pi$ we have $m\left(\pi^{\prime}\right)<m(\pi)$. For each partition $\lambda$ of $n$ choose a minimal sub-partition $\pi$ with $m(\pi)=m(\lambda)$ and write $\lambda=\pi \cup \omega$ for some partition $\omega$. Let $M_{n}$ be the set of all minimal partitions $\pi$ with $|\pi| \leq n$ and for any $\pi \in M_{n}$ let $\Omega_{\pi}$ be the set of all partitions $\omega$ that occur with $\pi$ in decompositions as above. Then

$$
\begin{aligned}
\mu_{n} & =\sum_{\pi \in M_{n}} \sum_{\omega \in \Omega_{\pi}} \frac{m(\pi)}{1^{c_{1}(\pi)} 2^{c_{2}(\pi)} \ldots 1^{c_{1}(\omega)} 2^{c_{2}(\omega)} \ldots c_{1}(\pi \cup \omega) ! c_{2}(\pi \cup \omega) ! \ldots} \\
& \leq \sum_{\pi \in M_{n}} \frac{m(\pi)}{\pi_{1} \pi_{2} \ldots} \sum_{\omega \in \Omega_{\pi}} \frac{1}{1^{c_{1}(\omega)} 2^{c_{2}(\omega)} \ldots c_{1}(\omega) ! c_{2}(\omega) ! \ldots} \\
& \leq \sum_{\pi \in M_{n}} \frac{m(\pi)}{\pi_{1} \pi_{2} \ldots}
\end{aligned}
$$

where the first inequality follows by rewriting $1^{c_{1}(\pi)} 2^{c_{2}(\pi)} \ldots$ as $\pi_{1} \pi_{2} \ldots$ and using $c_{i}(\pi \cup \omega) \geq c_{i}(\omega)$ and the second follows by noting that if the inner sum were over all partitions $\omega$ with $|\omega|=n-|\pi|$ instead of just a subset of them, then it would be 1 .

For each minimal $\pi=\left(\pi_{1}, \pi_{2}, \ldots, \pi_{s}\right)$ choose integers $\left(d_{1}, d_{2}, \ldots, d_{s}\right)$ with the following properties:

(1) $d_{i}$ divides $\pi_{i}$,

(2) g.c.d. $\left(d_{i}, d_{j}\right)=1$ for $i \neq j$,

(3) $\prod_{i=1}^{s} d_{i}=m(\pi)$.

(An explicit construction of the $d_{i}$ is given in [6].) Note that since $\pi$ is minimal the $d_{i}$ are all greater than 1 . Define integers $k_{i}$ by $\pi_{i}=k_{i} d_{i}$. Then $\pi_{1} \pi_{2} \ldots \pi_{s}=$ $m(\pi) k_{1} k_{2} \ldots k_{s}$. Let $D_{n}$ be the set of all unordered sets $(d)=\left(d_{1}, d_{2}, \ldots, d_{s}\right)$ of pairwise relatively prime integers greater than 1 with $d_{1}+d_{2}+\cdots+d_{s} \leq n$ and for any $(d) \in D_{n}$ let $K_{(d)}$ be the set of all $\left(k_{1}, k_{2}, \ldots, k_{s}\right)$ with $k_{1} d_{1}+k_{2} d_{2}+\cdots+k_{s} d_{s} \leq n$. Then the bound above becomes

$$
\mu_{n} \leq \sum_{(d) \in D_{n}} \sum_{(k) \in K_{(d)}} \frac{1}{k_{1} k_{2} \ldots} .
$$


The sets $\left(d_{1}, d_{2}, \ldots, d_{s}\right)$ can be broken up into two subsets: the prime elements and the composite elements. Any composite $d_{i}$ must be divisible by some prime $p$ with $p \leq \sqrt{n}$ and since the $d_{i}$ are relatively prime $p$ divides only one element. Therefore there are at most $\pi(\sqrt{n})<C \frac{\sqrt{n}}{\log n}$ composite elements. Each composite element contributes at most $\sum_{k=1}^{n} \frac{1}{k}=\log n+\mathbf{O}(1)$. Therefore all the composite elements together contribute at most $\exp \left\{\mathbf{O}\left(\frac{\sqrt{n} \log \log n}{\log n}\right)\right\}$ to $\mu_{n}$. Let $P_{n}$ be the set of all unordered sets $(d)=\left(d_{1}, d_{2}, \ldots, d_{s}\right)$ of distinct primes with $d_{1}+d_{2}+\cdots+d_{s} \leq n$. Then the bound above becomes

$$
\mu_{n} \leq \sum_{(d) \in P_{n}} \sum_{(k) \in K_{(d)}} \frac{1}{k_{1} k_{2} \ldots} \exp \left\{\mathbf{O}\left(\frac{\sqrt{n} \log \log n}{\log n}\right)\right\} .
$$

The sum above can be rewritten in a convenient form. Let $p_{1}, p_{2}, \ldots$ be all the primes in order and consider infinite sequences $\left(k_{1}, k_{2}, \ldots\right)$ with only finitely many nonzero terms with $\sum_{i=1}^{\infty} k_{i} p_{i} \leq n$. Then the sum above is the sum over all such sequences of the product of the reciprocals of the nonzero $k_{i}$ 's. Explicitly

$$
\mu_{n} \leq \sum_{(k): \Sigma k_{i} p_{i} \leq n} \prod_{i: k_{i} \neq 0} \frac{1}{k_{i}} \exp \left\{\mathbf{O}\left(\frac{\sqrt{n} \log \log n}{\log n}\right)\right\}
$$

If we define a function $h(t)$ and a sequence $a_{m}$ by

$$
h(t)=\prod_{p \text { prime }}\left(1-\log \left(1-e^{-p t}\right)\right)=\sum_{m=0}^{\infty} a_{m} e^{-m t},
$$

then the bound above says that

$$
\mu_{n} \leq \sum_{m=0}^{n} a_{m} \exp \left\{\mathbf{O}\left(\frac{\sqrt{n} \log \log n}{\log n}\right)\right\}
$$

Before analyzing the $a_{m}$ 's in detail we will first derive a lower bound comparable to this upper bound.

\section{The Lower Bound.}

Consider only partitions $\lambda$ of $n$ of the following nice form $\lambda=\pi \cup \omega$ where $\pi=\left(\pi_{1}, \pi_{2}, \ldots, \pi_{s}\right)$ and each $\pi_{i}=k_{i} d_{i}$ where the $d_{i}$ are distinct primes greater than $\sqrt{n}$ and $|\omega|<q$ where $q$ is the smallest prime larger than $\sqrt{n}$. For such a $\lambda$ we have $m(\lambda) \geq d_{1} d_{2} \ldots d_{s}$ and for all $i$ either $c_{i}(\lambda)=c_{i}(\omega)$ or $c_{i}(\lambda)=1$ and $c_{i}(\omega)=0$. In either case $c_{i}(\lambda) !=c_{i}(\omega)$ !. Therefore taking only the terms corresponding to these $\lambda$ 's in our expression for $\mu_{n}$ above gives

$$
\mu_{n} \geq \sum_{\pi} \frac{1}{k_{1} k_{2} \ldots} \sum_{\omega} \frac{1}{1^{c_{1}(\omega)} 2^{c_{2}(\omega)} \ldots c_{1}(\omega) ! c_{2}(\omega) ! \ldots}=\sum_{\pi} \frac{1}{k_{1} k_{2} \ldots}
$$


where the outer sum runs over all $\pi$ which occur in some partition as above and the second equality follows by noting that the inner sum is over all partitions $\omega$ with $|\omega|=n-|\pi|$ and hence is 1 . This lower bound can be rewritten as we did for the upper bound. Let $q=q_{1}<q_{2}<\ldots$ be all the primes greater than $\sqrt{n}$ in order and consider all infinite sequences $\left(k_{1}, k_{2}, \ldots\right)$ with only finitely many terms nonzero such that $n-q<\sum_{i=1}^{\infty} k_{i} q_{i} \leq n$. Then as above the lower bound is the sum over all such sequences of the product of the reciprocals of the nonzero $k_{i}$ 's. Define a functions $z_{n}(t)$ and sequences $b_{m}^{(n)}$ by

$$
z_{n}(t)=\prod_{p>\sqrt{n} \text { prime }} 1-\log \left(1-e^{-p t}\right)=\sum_{m=0}^{\infty} b_{m}^{(n)} e^{-m t}
$$

Then the lower bound above becomes

$$
\mu_{n} \geq \sum_{m=n-q+1}^{n} b_{m}^{(n)}
$$

We need only relate the $b_{m}^{(n)}$ to the $a_{m}$ defined earlier. Unfortunately the sum above extends over only a short range of indices; we must first correct this imbalance.

For any $m \leq n-q$ and any prime $q_{i}$ greater than $\sqrt{n}$ and any sequence $(k)$ that contributes to $b_{m}^{(n)}$ we obtain a sequence that contributes to $b_{m+q_{i}}^{(n)}$ by adding one to $k_{i}$. In the worst case this changes $k_{i}$ from 1 to 2 and halves the contribution of this term. Therefore $b_{m}^{(n)} \leq 2 b_{m+q_{i}}^{(n)}$. Since there is a prime $p$ between $(n-m) / 2$ and $n-m$ (which we may assume is greater than $\sqrt{n}$ since we may always take $p=q$ ) we may halve the distance from $m$ to $n$ by one application of this inequality. After at $\operatorname{most} \log _{2} n$ applications of the above inequality we obtain $b_{m}^{(n)} \leq n b_{s}^{(n)}$ for some $n-q<s \leq n$. Therefore we have

$$
\sum_{m=0}^{n} b_{m}^{(n)} \leq n^{2} \sum_{m=n-q+1}^{n} b_{m}^{(n)}
$$

therefore with only negligible error we may replace the sum in the lower bound above by the sum over all $m \leq n$.

To compare this sequence to the $a_{m}$ 's note that

$$
h(t)=z_{n}(t) \prod_{p \leq \sqrt{n} \text { prime }}\left(1-\log \left(1-e^{-p t}\right)\right) .
$$

If the second factor on the right hand side is expanded as $\sum_{m=0}^{\infty} c_{m}^{(n)} e^{-m t}$, then

$$
a_{m}=\sum_{k=0}^{m} b_{k}^{(n)} c_{m-k}^{(n)}
$$


The second factor of $h(t)$ is a product of $\pi(\sqrt{n})<C \frac{\sqrt{n}}{\log n}$ terms each of which contributes at most $1+\sum_{k=1}^{m} \frac{1}{k}=\log m+\mathbf{O}(1)$ to $c_{m}^{(n)}$. Therefore for all $m \leq n$ we see $c_{m}^{(n)} \leq \exp \left\{\mathbf{O}\left(\frac{\sqrt{n} \log \log n}{\log n}\right)\right\}$, so $a_{m} \leq \sum_{k=0}^{m} b_{k}^{(n)} \exp \left\{\mathbf{O}\left(\frac{\sqrt{n} \log \log n}{\log n}\right)\right\}$. Summing over $m$ gives

$$
\mu_{n} \geq \frac{1}{n^{2}} \sum_{m=0}^{n} b_{m}^{(n)} \geq \sum_{m=0}^{n} a_{m} \exp \left\{-\mathbf{O}\left(\frac{\sqrt{n} \log \log n}{\log n}\right)\right\} .
$$

Combining this with the upper bound above gives

$$
\log \mu_{n}=\log \sum_{m=0}^{n} a_{m}+\mathbf{O}\left(\frac{\sqrt{n} \log \log n}{\log n}\right) .
$$

To complete the proof we need only bound $\log \sum_{m=0}^{n} a_{m}$.

\section{The Tauberian Theorem.}

We will apply the following result of Erdös and Turán [3].

Lemma (Erdős and Turán) Let $f(t)=\sum_{m=0}^{\infty} a_{m} e^{-m t}$ and suppose

$$
\log f(t)=\frac{A}{t \log 1 / t}+\mathbf{O}\left(\frac{\log \log 1 / t}{t(\log 1 / t)^{2}}\right) \quad \text { as } t \rightarrow 0^{+} .
$$

Then

$$
\sum_{m=0}^{n} a_{m}=\exp \left\{2 \sqrt{2 A \frac{n}{\log n}}+\mathbf{O}\left(\frac{\sqrt{n} \log \log n}{\log n}\right)\right\} .
$$

Thus we need only analyze $\log h(t)$ as $t \rightarrow 0^{+}$. As in [3] we have

$$
\begin{aligned}
\log h(t) & =\sum_{p \text { prime }} \log \left(1-\log \left(1-e^{-p t}\right)\right)=\int_{0}^{\infty} \log \left(1-\log \left(1-e^{-x t}\right)\right) d \pi(x), \\
& =\int_{0}^{\infty} \frac{t \pi(x) e^{-x t}}{\left(1-e^{-x t}\right)\left(1-\log \left(1-e^{-x t}\right)\right)} d x \\
& =\int_{0}^{\infty} \frac{\pi(s / t) e^{-s}}{\left(1-e^{-s}\right)\left(1-\log \left(1-e^{-s}\right)\right)} d s
\end{aligned}
$$

The integrand is bounded by $C_{1} t^{-1}$ for $s$ small (using the bound $\pi(x) \leq x$ ). Therefore the contribution to the integral from the interval $\left[0, t^{1 / 2}\right)$ is bounded by $C_{1} t^{-1 / 2}$. Hence we may replace the lower endpoint by $t^{1 / 2}$ with only a negligible error. For any $x$ we have

$$
\pi(x)=\frac{x}{\log x}+\mathbf{O}\left(\frac{x}{(\log x)^{2}}\right),
$$

(see for example [5, Thm 23, p. 65]) hence

$$
\pi(s, t)=\frac{1}{t} \frac{s}{\log 1 / t+\log s}+\mathbf{O}\left(\frac{1}{t} \frac{s}{(\log 1 / t+\log s)^{2}}\right) .
$$


Since $s \geq t^{1 / 2}$ we have $\log s \geq-1 / 2 \log (1 / t)$ and thus

$$
\begin{aligned}
\pi(s, t) & =\frac{s}{t \log 1 / t}-\frac{s \log s}{t \log 1 / t(\log 1 / t+\log s}+\mathbf{O}\left(\frac{s}{t(\log 1 / t)^{2}}\right) \\
& =\frac{s}{t \log 1 / t}+\mathbf{O}\left(\frac{s(1+|\log s|)}{t(\log 1 / t)^{2}}\right)
\end{aligned}
$$

Plugging this into the integral and extending the lower endpoint back to 0 (which again introduces only negligible error terms) gives

$$
\log h(t)=\frac{1}{t \log 1 / t} \int_{0}^{\infty} \frac{s e^{-s}}{\left(1-e^{-s}\right)\left(1-\log \left(e^{-s}\right)\right)} d s+\mathbf{O}\left(\frac{1}{t(\log 1 / t)^{2}}\right) .
$$

So the Tauberian theorem of Erdős and Turán gives

$$
\log \mu_{n}=2 \sqrt{2 A} \sqrt{\frac{n}{\log n}}+\mathbf{O}\left(\frac{\sqrt{n} \log \log n}{\log n}\right)
$$

where

$$
\begin{aligned}
A & =\int_{0}^{\infty} \frac{s e^{-s}}{\left(1-e^{-s}\right)\left(1-\log \left(1-e^{-s}\right)\right)} d s=\int_{0}^{\infty} \frac{\log (s+1)}{e^{-s}-1} d s \\
& =\sum_{n=1}^{\infty} \frac{e^{n}}{n} E_{1}(n)=1.11786415 \ldots
\end{aligned}
$$

where $E_{1}(n)$ is the exponential integral (see [1, Eqn. 5.1.1, p. 228]).

Acknowledgements. The author was partially supported by an Alfred P. Sloan Research Fellowship.

\section{REFERENCES}

1. M. Abramowitz and I. A. Stegun, Handbook of Mathematical Functions, National Bureau of Standards, Washington DC, 1972.

2. P. Erdős and P. Turán, On some problems of a statistical group theory, III, Acta Math. Acad. Sci. Hungar. 18 (1967), 309-320.

3. P. Erdős and P. Turán, On some problems of a statistical group theory, IV, Acta Math. Acad. Sci. Hungar. 19 (1968), 413-435.

4. W. Goh and E. Schmutz, The expected order of a random permutation, Bull. Lond. Math. Soc. 23 (1991), 34-42.

5. A. E. Ingham, The Distribution of Prime Numbers, Cambridge University Press, Cambridge, 1990.

6. E. Schmutz, Proof of a conjecture of Erdős and Turán, Jour. No. Th. 31 (1989), 260-271. 